\title{
Neurological Worsening in a Child of Miliary Tuberculosis with Neuro-Tuberculosis on Anti Tubercular Treatment
}

\author{
Arora $\mathrm{S}^{1}$, Narang $\mathrm{GS}^{2}$, Dhillon $\mathrm{PK}^{3}$
}

\begin{abstract}
A seven and half year old male child presenting with pyrexia of unknown origin was diagnosed to be a case of miliary tuberculosis. Neuroimaging revealed multiple discrete ring as well as nodular enhancing lesions indicative of tuberculomas. After the initial response to ATT along with systemic steroids the child again presented with severe headache along with vomiting towards the end of intensive phase. Repeat neuroimaging showed appearance of new lesions with perilesional edema. Child was started again on systemic steroids and Streptomycin was added to the anti tubercular regimen, to which the child responded well.
\end{abstract}

Key words: Miliary tuberclosis, Tuberculoma, Paradoxical reaction

\section{Introduction}

C NS tuberculosis remains an important cause of mortality and morbidity in developing countries with resurgence in developed countries due to appearance of AIDS. Many of the symptoms, signs and sequelae of neurotuberculosis are the result of an immunologically directed inflammatory reaction to the infection ${ }^{1}$. Bacilli implanted on the meninges or brain parenchyma, result in the formation of small lesions (Rich foci). The location of the expanding Rich focus determines the type of involvement. Tubercles rupturing into the subarachnoid space cause meningitis. Those deeper in the brain parenchyma cause tuberculomas. Arachnoiditis and infarcts are other features of neurotuberclosis ${ }^{2,3}$. While these lesions usually resolve following antitubercular therapy (ATT), rarely clinical deterioration may occur in spite of good initial recovery. This worsening in neuro-tuberculosis has been attributed to a paradoxical response and may occur within days and even one year after starting standard anti tubercular chemotherapy $y^{4,5}$. Paradoxical response is defined as recurrence or appearance of fresh symptoms, physical and radiological signs in a patient who had previously shown improvement with appropriate anitubercular treatment. The present case presented with features of raised intracranial pressure with appearance of new lesions in brain towards end of intensive phase of antitubercular treatment.
'Dr. Sunita Arora, ${ }^{2}$ Dr. Gursharan Singh Narang, Professor Paediatrics, ${ }^{3}$ Dr. Prabhjot Kaur Dhillon, Junior Resident. All from the Department of Paediatrics, Sri Guru Ram Das Institute of Medical Sciences and Research,Vallah, Sri Amritsar.

\section{Address for correspondence: \\ Dr. Gursharan Singh Narang \\ E-mail: gsnarang321@gmail.com}

\section{How to cite}

Arora S, Narang GS, Dhillon PK. Neurological Worsening in a Child of Miliary Tuberculosis with Neuro-Tuberculosis on Anti Tubercular Treatment. J Nepal Paediatr Soc 2015;35(2):185-188.

doi: http://dx.doi.org/10.3126/jnps.v35i2.10406

This work is licensed under a Creative Commons Attribution 3.0 License.

\section{(c) (7)}

\section{The Case}

A seven and a half year old male child presented to us with complaints of high grade fever for six weeks followed by frontal headache and non projectile non bilious vomiting off and on with decreased appetite since past seven days. There was no history of cough, urinary complaints, rigor and chills, altered sensorium, neurological deficit or seizures during the course of illness. $\mathrm{He}$ had received treatment for enteric fever and Malaria by private practitioners but without any response. History of pulmonary tuberculosis was elicited in grandfather 5 years back. On examination child was conscious, well oriented to time, place and person. Vital parameters were within normal range. Child had mild hepato splenomeagly with no significant 
lymphadenopathy. Chest examination was also normal. Meningeal signs were absent. Rest of the neurological examination was normal. Fundus examination showed multiple choroid tubercles. CBC, Urine analysis, blood culture and Widal agglutination test were non contributory. Tuberculin test was non reactive. ESR was raised (60 $\mathrm{mm} 1^{\text {st }}$ hour). HIV status was negative. CSF examination was not done. CXR showed miliary tuberculosis (Figure 1). MRI Brain revealed multiple predominantly discrete ring as well nodular enhancing intra axial lesions suggestive of tuberculomas (Figure 2). Diagnosis of Miliary TB with neurotuberculosis was made. He was started on $2 \mathrm{HRZE}+10 \mathrm{HR}$ along with systemic steroids for six weeks.Child improved clinically and remained well for about 6-7 weeks. Towards the end of intensive phase there was recurrence of severe

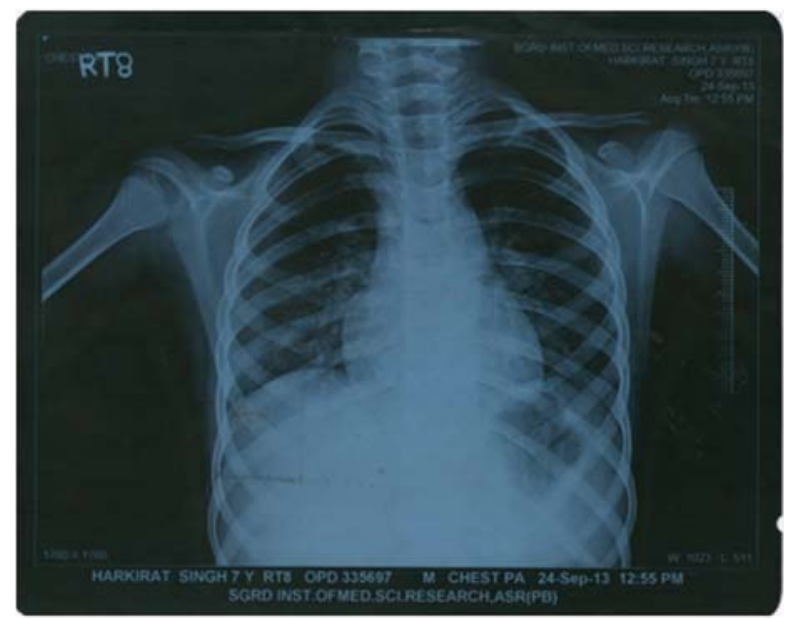

Fig 1: $X$ ray chest PA view showing military mottling

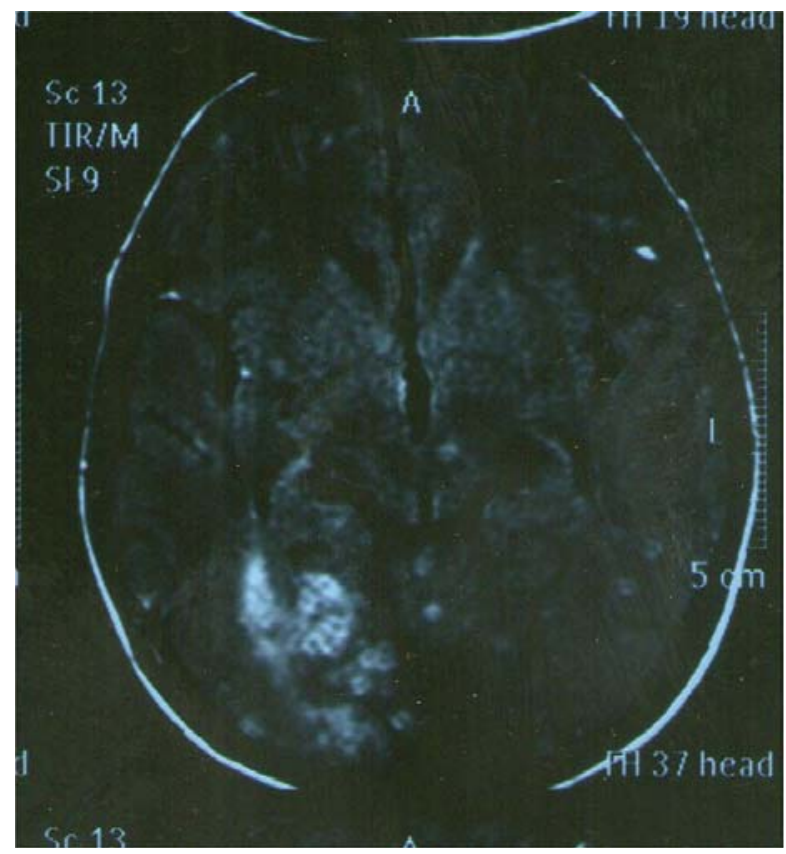

Fig 2: MRI brain axial section flair sequence showing scattered hyperintensities. headache along with vomiting for 4-5 days. His vitals were within normal limits and neurological examination was normal. Repeat MRI brain showed appearance of new ring enhancing lesions with perilesional edema (Figure 3). Fundus examination was normal. Diagnosis of paradoxical immune response to ATT was made and child was restarted on systemic steroids. The intensive phase for ATT was extended for another two months and injection Streptomycin was added to the regimen. In addition child was also given intravenous Mannitol injection for two days to relieve cerebral oedema. The child showed improvement after 72 hours and remained well thereafter. CXR also revealed resolution of military tuberculosis. MRI repeated at six months revealed complete resolution except few calcified lesions in cerebellum.

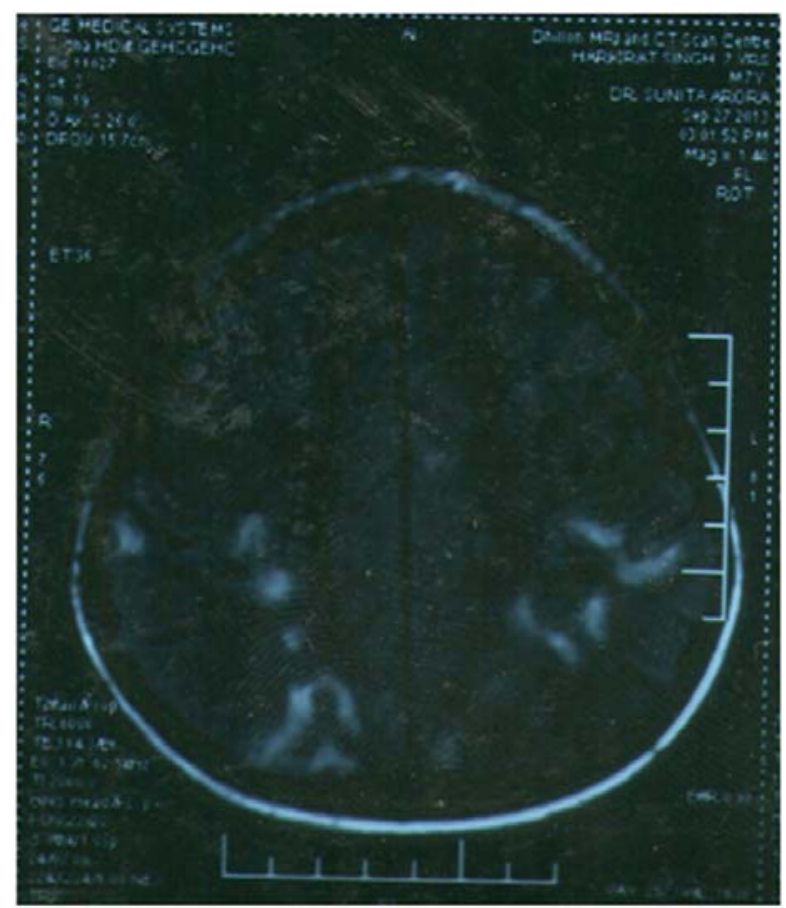

Fig 3: New ring enhancing lesions with perilesional oedema (after 2 months of intensive phase of ATT).

\section{Discussion}

Paradoxical response is now increasingly being recognized as a cause of subsequent deterioration in cases of CNS tuberculosis despite adequate and appropriate therapy. This phenomenon complicates the decision about the therapy of CNS tuberculosis. It is not possible to clearly differentiate between paradoxical deterioration and development of secondary resistance in the absence of positive tests of culture and sensitivity for Mycobacterium tuberculosis. Expansion of a tuberculoma or development of multiple new brain lesions during treatment of 
TBM, though uncommon, has been reported in the literature and is called a paradoxical response $e^{6,7,8,9,10}$. Meena Gupta et al described that the duration of time between initiation of therapy and worsening of patient was from one to seven months. Nine out of ten patients developed fresh intracranial tuberculoma while one case otherwise showing improvement developed expansion of tuberculoma and other one of tubercular empyema developed tuberculoma while on therapy. All these cases responded to addition of second line drug or increase in dose of drugs previously prescribed and introduction or increased dose of steroid $^{8}$. Ajay SK et al described the occurrence of this condition, though previously reported only in the developing world, is now frequently reported in human immunodeficiency virus positive migrants in the western world. The exact pathogenesis of this condition is still incompletely understood, and the mainstay of treatment is chemotherapeutic regimes along with systemic steroids. Neurosurgical intervention is rarely necessary, and is confined to cases of hydrocephalus after tubercular meningitis and to large tubeculomas with space-occupying effects ${ }^{12}$.

The explanation for paradoxical response to treatment remains unclear. Various hypotheses have been put forward to explain this unusual phenomenon. One possibility is that this occurs because of decreased penetration of antitubercular drugs into brain ${ }^{12}$. Restoration of blood brain barrier with appropriate treatment is proposed to result in reactivation of latent foci. However, this hypothesis cannot explain the development or enlargement of intracranial tuberculoma who are treated with isoniazid and pyrazinamide, both of which freely cross the blood brain barrier in the absence of inflamed meninges. This does not explain the improvement observed in patients in whom significant change is made in antitubercular therapy. Enlargement of lymph nodes (which do not have the barrier like blood brain barrier) in patients on anti-tubercular therapy further goes against the hypothesis. Paradoxical response is possibly due to a Type IV hypersensitivity reaction developing within the initial lesion and resulting in cerebral vasculitis, infarction, and edema ${ }^{11,12,13}$. Paradoxical reactions occur due to complex interplay between host's immune response and the direct effect of mycobacterial antigens ${ }^{14,15,16}$. Active tuberculosis leads to depression of type IV hypersensitivity reaction and immunosuppression due to activation of monocytes by protein derivatives of mycobacteria. Increased interleukin levels resulting from this can lead to immunosuppression. Specific antigens lead to production of immunosuppressive concentration of
prostaglandin-E2 ${ }^{8}$. Once active tuberculosis is under control after starting ATT, immunosuppression resolves. It also leads to enhanced delayed-type hypersensitivity and activation and accumulation of lymphocytes and macrophages at the site of bacterial deposition or toxin production occurs when bacilli die. The reason for occurrence of this response in only some cases and not all suggests that it depends on host immune responses, virulence of tubercle bacilli, antigen load and effective antitubercular therapy. The yield of AFB culture from CSF can be increased by centrifuging the CSF, preparing thick smears from cobweb and increasing the examination time. The solution to this dilemma of paradoxical response with negative laboratory support, lies in close monitoring of patient with continuation of drugs already in use with addition of steroid, increasing the dose of drugs already in use and/or addition of second line $\mathrm{ATT}^{17,18,19,20}$.

\section{Conclusion}

In the final analysis, clinical judgement, regular follow up, guarded reassurance of patient and recognition of the possibility of paradoxical response is the only practical answer. There is also a need to report their occurrence so as to chronicle the entire spectrum.

\section{References}

1. Dastur DK, Manghani DK, Udani PM. Pathology and pathogenetic mechanisms in neurotuberculosis. Radiol Clin North Am 1995;33:733-52.

2. Kalita J, Misra UK. Effect of methyl prednisolone on sensory motor functions in tuberculous meningitis. Neurol India 2001;49:267-71.

3. Gupta RK, Gupta S, Singh D, Sharma B, Kohli A, Gujral RB. MR imaging and angiography in tuberculous meningitis. Neuroradiology 1994;36:87-92.

4. Lees AJ, McLeod AF, Marshall J. Cerebral tuberculoma developing during treatment of tuberculous meningitis. Lancet 1980;1:1208-211.

5. Kumar R, Prakash M, Jha S. Paradoxical response to chemotherapy in neurotuberculosis. Pediatr Neurosurg 2006;42:214-22.

6. Safdar A, Brown AE, Kraus DH, Malkin M. Paradoxical reaction syndrome complicating aural infection due to mycobacterium tuberculosis during therapy. Clin Infect Dis 2000;30:625-27.

7. Reiser M, Fatkenheuer G, Diehl V. Paradoxical expansion of intracranial tuberculomas during chemotherapy. J Infect 1997;35:88-90. 
8. Gupta M, Bajaj BK, Khwaja G. Paradoxical response in patients with CNS tuberculosis. J Assoc Physicians India 2003;51:257-60.

9. Kalkan A, Serhatlioglu S, Ozden M, Denk A, Demirdag K, Yilmaz T, et al. Paradoxically developed optochiasmatic tuberculoma and tuberculous lymphadenitis: A case report with 18-month follow up by MRI. South Med J 2006;99:388-92.

10. PauranikA, Behari M, Maheshwari MC. Appearance of tuberculomas during treatment of tuberculous meningitis. Jpn J Med 1987;26:332-34.

11. Malik GM, Basu JA, Hussain T. Paradoxical expansion of cerebral tuberculomas during therapy for Pott's spine. J R Soc Med 1996;89:643-44.

12. Ajay SK, Lakhkar BB, Bhaskaranand N. Intracranial tuberculoma manifesting during treatment. Indian Paediatr 1996;33:231-33.

13. Parveen K Monga and Upreet DhaliwalParadoxical reaction in tubercular meningitis resulting in involvement of optic radiation. Indian J Ophthalmol 2009;57(2):139-41.

14. Chambers ST, Hendrickse WA, Record C, Rudge $P$, Smith H.Paradoxical expansion of intracranial tuberculomas during chemotherapy. Lancet 1984;2:181-84.
15. Marshall BG, Chambers MA. Central nervous systemtuberculosis - the paradox of the host immune response. J Infect 1998;36:3-4

16. Van Bommel EF, Stiegelis WF, Schermers HP. Paradoxicalresponse of intracranial tuberculomas during chemotherapy:an immunologic phenomenon? Neth J Med 1991;38:126-30.

17. Reser M, Fatkenheur G, Diehl V. Paradoxical expansion of intracranial tuberculomas during the chemotherapy. J Infect 1997;35:88-90.

18. BukharieH.Paradoxical responsetoantituberculous drugs: resolution with corticosteroid therapy. Scan J Infect Dis 2000;32:96-7.

19. Rao GP, Nadh BR, Hemaratnan A, Srinivas TV, Reddy PK. Paradoxical progression of tuberculous lesions during chemotherapy of central nervous system tuberculosis. Report of four cases. J Neurosurg 1995;83:359-62.

20. Abdul Jabbar M. Paradoxical response to chemotherapy for Inracranial tuberculoma: two case reports from Saudi Arabia. J Trop Med Hyg 1991;94:374-76. 